\title{
Investigating Distribution of Capacity Based on Degradation Model
}

\author{
Chang-qiao Shao and Xiao-ming Liu \\ Beijing Key Lab of Transportation Engineering, Beijing University of Technology, Beijing 100124, China \\ Correspondence should be addressed to Chang-qiao Shao; shaocq@bjut.edu.cn
}

Received 27 June 2014; Revised 27 September 2014; Accepted 27 September 2014

Academic Editor: Wuhong Wang

Copyright ( 2015 C.-q. Shao and X.-m. Liu. This is an open access article distributed under the Creative Commons Attribution License, which permits unrestricted use, distribution, and reproduction in any medium, provided the original work is properly cited.

The breakdown phenomenon at freeway has both practical and theoretical interests. Many researchers have done great efforts on it. As a result, the stochastic concept for freeway capacity was defined and some approaches were developed. However, due to the fact that it is impossible to observe the value of the capacity, it is difficult to obtain the exact traffic capacity distribution. In this paper, traffic operational degradation concept was introduced and degradation model was developed to describe traffic breakdown process. A stochastic degradation parameter is introduced to explain the phenomena of traffic breakdown occurring at various flow rate levels. Traffic breakdown probability and traffic capacity distribution were derived theoretically. Based on the surveyed data, the experimental model was obtained by using the bootstrap method. The results indicate that Weibull distribution function can be used to describe traffic capacity stochastic nature.

\section{Introduction}

In the last decades, the capacity of a freeway section is most taken as a constant value. For example, in the current published Highway Capacity Manual (HCM2000) [1], it was still defined as the maximum hourly rate at which vehicles reasonably can be expected to traverse a point or a uniform section of a lane or roadway during a given time period under prevailing roadway, traffic, and control conditions. However, more and more investigations [2-12] showed that the traditional definition of capacity cannot explain the traffic phenomena such as traffic breakdown occurring at various flow rate levels. In theory, if the demand exceeds capacity, breakdown will occur. However, more and more researches show that traffic flow presents complexity and that the breakdown has probability nature. In order to describe this phenomenon, different methods are employed. For example, Kerner [3] developed three phase traffic theory to explore traffic breakdown features and highway capacity stochastic nature. Freund et al. $[13,14]$ applied statistical physic theory and introduced the phase transition concept to describe the breakdown phenomenon.
In this paper, traffic breakdown was studied in a new view and it is considered as the result of traffic operational degradation. Analogous to electronic product degradation, if the traffic speed drops to some threshold value, it is called traffic operational degradation. Based on this concept, breakdown probability and capacity distribution model are developed.

This paper is organized as follows. In Section 2, we present a brief literature review. In Section 3, the traffic degradation model is developed. In Section 4, the breakdown and capacity distribution models are derived. In Section 5, we give the empirical analysis results. Section 6 presents the summary.

\section{Literature Review}

In order to understand the freeway capacity, researchers have made great efforts to model and describe stochastic nature of freeway capacity and breakdown phenomenon. Polus et al. $[2,3]$ studied the stochastic nature of freeway capacity and used Gamma distribution to describe the capacity. But the definition of capacity is still based on the maximum 
flow rates. Zhang and David [8] examined the process of breakdown in detail and noted that the current HCM freeway capacity definition does not accurately reflect the relationship between breakdown and flow rate. They also suggested that freeway capacity should be described by incorporating a probability of breakdown component in the definition. Lorenz and Elefteriadou [9] introduced a new understanding of freeway capacity as the traffic flow below which traffic still flows and above which the traffic breaks down. In their study, a traffic breakdown is regarded as a failure event and the Product Limit Method for lifetime data analysis was used to estimate the capacity. Brilon et al. [10] studied the breakdown flow rates. It was found that breakdown may occur at flows lower than the capacity and that breakdown is a probabilistic event. A linear model for describing the process of breakdown at ramp-freeway junction was developed. Ozbay and Ozguven [11] examined the breakdown flow, prebreakdown flow, and maximum discharge flow to determine which one would be more appropriate for use in defining the "capacity" of a freeway. It was recommended that the breakdown flow be used. But they also pointed out that the numerical value of each of these three parameters varies and their ranges are relatively large, in the order of several hundred veh/h/lane. Biham et al. [12] provided the parameter estimating method for capacity probability distribution. It was concluded that including random component in the model results in better representation of observed data and thus improves understanding of real-life situation. Freund and Pöschel [13] analyzed traffic volume time series for dense traffic. They showed that traffic flow will decay over time and unstable traffic flow where a breakdown can be expected with certainty. Nagel and Paczuski [15] studied a single-lane traffic model using simulation and it is observed numerically that traffic breakdown occurs at or near the point of maximum throughput and that the flow behavior in this region is complex.

From these efforts, it is concluded that the breakdown phenomenon should be considered in the definition of traffic capacity. Due to the fact that the breakdown flows vary greatly, the traditional definition of freeway capacity is not proper and the definition based on probability distribution should be the better one.

\section{Traffic Degradation and Breakdown}

According to traffic theory $[14,16,17]$, traffic velocity for freeway will decrease with increasing of volume. Figure 1 shows a typical speed-flow diagram obtained from empirical data. From Figure 1, it is easily found that when the traffic flow exceeds some threshold, the traffic will fall into congestion (i.e., traffic breakdown occurs). In the field of lifetime analysis, if the electronic product does not work well, it is called degradation [18]. Analogously, we call the traffic breakdown phenomena as traffic operational degradation. Similarly, we use the traffic speed as the freeway traffic performance measure which will fall with increasing of traffic

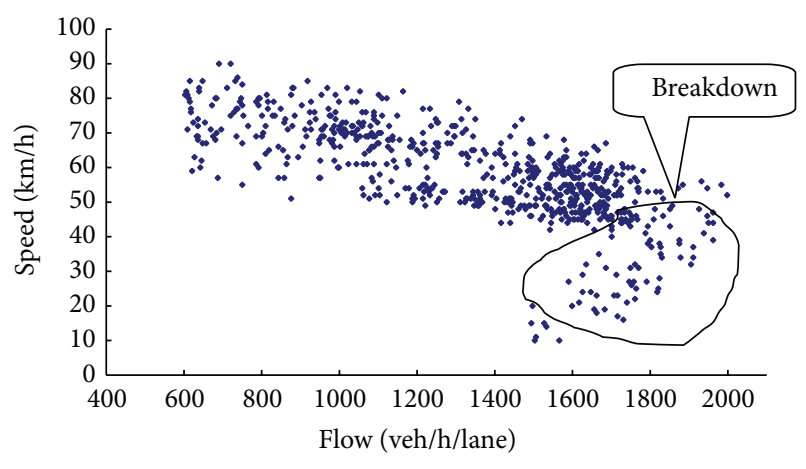

Figure 1: Speed-flow plot of freeway section.

volume just as the electronic product degenerates with time. Then the traffic degradation model is

$$
V=D\left(q, \beta_{1}, \ldots, \beta_{k}\right)+\varepsilon_{q}
$$

where $V$ is speed, $\mathrm{km} / \mathrm{h} ; q$ is flow rate, veh/h/lane; $D\left(q, \beta_{1}\right.$, $\left.\ldots, \beta_{k}\right)$ is the function of $q ; \beta_{1}, \ldots, \beta_{k}$ are parameters; and $\varepsilon_{q}$ is the random error. Due to traffic fluctuation (such as a random increase of traffic and unexpected vehicle deceleration), $D\left(q, \beta_{1}, \ldots, \beta_{k}\right)$ is also taken as random variable (Figure 2 presents the observed degradation of traffic operation in peak time for one week; it shows randomness of $\left.D\left(q, \beta_{1}, \ldots, \beta_{k}\right)\right)$. The randomness is due to two kinds of traffic fluctuation. One is the different behavioral patterns of the individual drivers, such as an abrupt deceleration which leads to a local decrease in speed and overreaction while slowing down. The second kind of fluctuation is hysteresis effects $[12,13]$. The evidence is that the breakdown occurs at different flow rates [10]. $\varepsilon_{q}$ is random error (which can be taken as observation error due to the time interval and the different behavior of the drivers. For example, when the traffic is free-flow, $\varepsilon_{q}$ represents the difference of driver's speed choice), and it is assumed that $\varepsilon_{q}$ follows normal distribution, $\varepsilon_{q} \sim N\left(0, \sigma^{2}\right)$. Furthermore, $d V / d q=D^{\prime}\left(q, \beta_{1}, \ldots, \beta_{k}\right)$ is called degradation rate. It is supposed that some of the parameters among $\beta_{1}, \ldots, \beta_{k}$ are deterministic and others are random. The deterministic parameters reflect the nonstochastic characteristic of the traffic degradation and the random parameters represent the complexity of traffic degradation (such as nonlinearities between input of volume and output of velocity). It is also supposed that the error term $\varepsilon_{q}$ and the parameters $\beta_{1}, \ldots, \beta_{k}$ are independent.

In model (1), the degradation function $D\left(q, \beta_{1}, \ldots, \beta_{k}\right)$ and the distributions of $\beta_{1}, \ldots, \beta_{k}$ are the keys to determine the traffic degradation model. In Highway Capacity Manual [1], $D\left(q, \beta_{1}, \ldots, \beta_{k}\right)$ has two segments; one part is horizontal and the other part is smooth curve (see Figure 3, Figure 1 also presents this characteristic). From both Figures 1 and 3 , it is concluded that when the traffic demand exceeds some threshold, the traffic operational performance begins to degrade (velocity decreases quickly) with increasing of traffic 


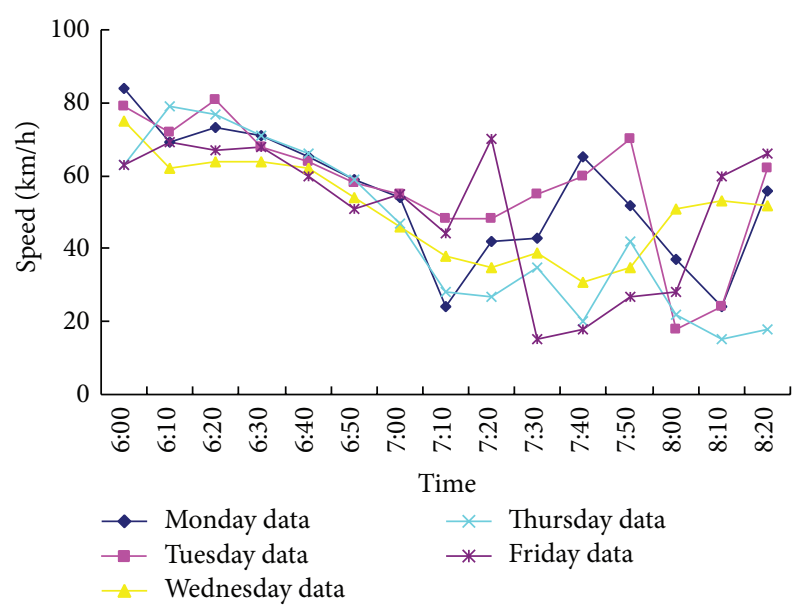

FIgURE 2: Observed operational degradation of traffic performance.

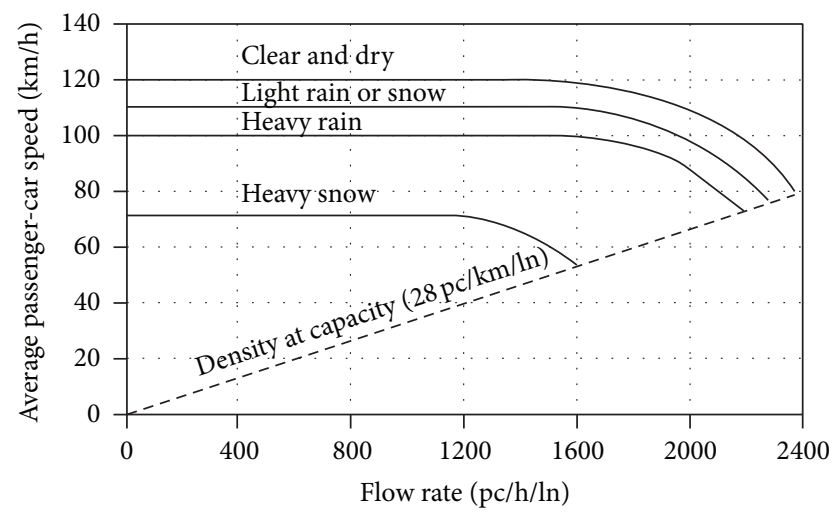

Figure 3: Speed-flow curves presented in Highway Capacity Manual.

demand and that the degradation rate is proximately linear. Therefore, the degradation model is supposed as

$$
D\left(q, \beta_{1}, \beta_{2}\right)= \begin{cases}\beta_{1} & q<q_{d} \\ \beta_{1}-\beta_{2}\left(q-q_{d}\right) & q \geq q_{d}\end{cases}
$$

where $\beta_{1}$ is constant (i.e., free-flow speed); $q_{d}$ is the traffic flow threshold at which speed begins to decrease with increasing of flow. $\beta_{2}=-d D\left(q, \beta_{1}, \beta_{2}\right) / d q$ is degradation rate. In real life, due to the randomness of traffic fluctuation such as drivers' acceleration or deceleration behavior, $\beta_{2}$ is a random variable and has a distribution (Figure 2 illustrates that the speed drops with different slope). If the traffic fluctuation is acute, traffic speed will have a deep downturn, and $\beta_{2}$ will take bigger value. In this case, a smaller increasing of volume will cause speed to decrease substantially and traffic breakdown to occur. Otherwise, if fluctuation is smooth, $\beta_{2}$ will take smaller value and the traffic flow is more stable. Therefore, $\beta_{2}$ can be used to explain why traffic breakdown will occur at various flow rate levels.

From model (2), it can be explained that breakdown is mainly caused by increasing of flow rate and traffic perturbation. If the fluctuations and flow rate exceed some value (e.g., the flow exceeds road capacity), breakdown will happen. Otherwise, the traffic will operate smoothly.

\section{Traffic Breakdown Probability and Capacity Distribution}

The main characteristic of traffic breakdown is associated with an abrupt decrease in traffic flow speed $[19,20]$. Therefore, traffic breakdown also is called speed breakdown and can be described by drop of speed $[19,20]$. Let $v_{0}$ be denoted as the speed threshold; that is, if the traffic speed is observed lower than $v_{0}$, it indicates that traffic breakdown has occurred.

Using $\left\{V<v_{0}\right\}$ to denote breakdown event, which can be rewritten as $\left\{D\left(q, \beta_{1}, \beta_{2}\right)+\varepsilon_{q} \leq v_{0}\right\}$. And the breakdown probability is

$$
\begin{aligned}
P\left(V<v_{0}\right) & =\int_{-\infty}^{+\infty} P\left\{D\left(q, \beta_{1}, \beta_{2}\right)+\varepsilon_{q} \leq v_{0}\right\} d \varepsilon_{q} \\
& =P\left\{D\left(q, \beta_{1}, \beta_{2}\right) \leq v_{0}\right\} .
\end{aligned}
$$

Substitute (2) into (3); breakdown probability is calculated as

$$
P_{b}=P\left\{\beta_{2} \geq \frac{\left(\beta_{1}-v_{0}\right)}{\left(q-q_{d}\right)}\right\},
$$

where $P_{b}$ is the breakdown probability. $\beta_{1}, v_{0}$, and $q_{d}$ are parameters as above defined. Due to the fact that the flow $q$ reflects the effect of traffic demand on the traffic breakdown and degradation rate $\beta_{2}$ reflects the effect of traffic fluctuation, model (4) indicates that traffic flow $q$ and degradation rate $\beta_{2}$ are the main influence factors of traffic breakdown probability for a given freeway section.

For different distribution choice of $\beta_{2}$, distinct model can be derived. For example, if it is supposed that $\beta_{2}$ has an inverse Weibull distribution:

$$
F_{\beta_{2}}(b)=1-\exp \left\{-\left(\frac{1}{\eta b}\right)^{\alpha}\right\},
$$

where $\alpha$ is the shape parameter and $\eta$ is the scale parameter. The breakdown probability derived from (4) and (5) is Weibull distribution:

$$
P_{b}=1-\exp \left\{-\left[\frac{\left(q-q_{d}\right)}{\eta\left(\beta_{1}-v_{0}\right)}\right]^{\alpha}\right\} .
$$

In another view, the ultimate cause of traffic breakdown is that traffic demand exceeds facility capacity. It means that the event $\left\{V<v_{0}\right\}$ is equivalent to the event that traffic flow exceeds the capacity $C$; that is, $\{C<q\}$. So, according to the definition of capacity $[7,8]$, model (6) can be reexpressed as

$$
\begin{gathered}
P(C<q)=1-\exp \left\{-\left[\frac{\left(q-q_{d}\right)}{\eta\left(\beta_{1}-v_{0}\right)}\right]^{\alpha}\right\}, \\
F_{C}(q)=1-\exp \left\{-\left[\frac{\left(q-q_{d}\right)}{\eta\left(\beta_{1}-v_{0}\right)}\right]^{\alpha}\right\},
\end{gathered}
$$


TABLE 1: The goodness-of-fit test result for $\beta_{2}$.

\begin{tabular}{|c|c|c|c|c|c|}
\hline \multirow{2}{*}{ Fitted distribution } & \multicolumn{2}{|c|}{ Parameters } & \multicolumn{3}{|c|}{ Goodness-of-fit } \\
\hline & Scale parameter & Shape parameter & Chi-square & Degree of freedom & $P$ value \\
\hline Inverse Weibull distribution $^{\mathrm{a}}$ & 40.74 & 21.57 & 113.46 & 7 & 0.000 \\
\hline Log-normal distribution function ${ }^{\mathrm{b}}$ & -3.68 & 0.046 & 10.61 & 6 & 0.101 \\
\hline Normal distribution $^{c}$ & 39.86 & 1.83 & 10.61 & 7 & 0.294 \\
\hline
\end{tabular}

${ }^{a}$ Hypothesis testing is that the reciprocal value distribution is Weibull function; ${ }^{b}$ the hypothesis is that $\log$ value distribution is normal distribution; ${ }^{c}$ the hypothesis is that the reciprocal is normal distribution.

where $P(C<q)$ is the probability of capacity less than traffic flow $q ; F_{C}(q)$ is the distribution of capacity. It is noted that model (8) is Weibull distribution function with shape parameter $\alpha$ and scale parameter $\eta\left(v_{0}-\beta_{1}\right)$.

One can also suppose that $\beta_{2}$ follows the log-normal distribution:

$$
P\left(\beta_{2}<b\right)=\Phi\left(\frac{(\ln b-u)}{\sigma}\right)
$$

where $\Phi(\cdot)$ is the standard normal distribution function and $u$ and $\sigma^{2}$ are parameters. From (8) and (9), it can be derived that the capacity is also log-normal distributed:

$$
F_{C}(q)=\Phi\left(\frac{\ln \left(q-q_{d}\right)-\ln \left(v_{0}-\beta_{1}\right)-u}{\sigma}\right) \quad q>q_{d}
$$

Similarly, different distribution hypothesis can be made on the degradation rate $\beta_{2}$ and other models can be derived. In real world, the distribution of $\beta_{2}$ is unknown and it can be estimated using Bootstrap method [21].

\section{Empirical Analysis}

The field data were collected on the 3rd ring in Beijing City, China, by using microwave detectors. The posted speed limit on the $3 \mathrm{rd}$ ring is $80 \mathrm{~km} / \mathrm{h}$. Traffic breakdowns are typical during morning and afternoon peak periods. One-month data are used in this paper. Based on the speed-flow curve, $q_{d}=900$ vehicles per hour per lane and speed threshold value $v_{0}=40 \mathrm{~km} / \mathrm{h}$ are determined (see Figure 1 ).

Let $\left(q_{i}, v_{i}\right)(i=1, \ldots, n)$ denote the observed value of flow rate and speed. Due to the fact that the degradation is mainly concerned about the free flow, only the upper branch data are used. So, the data were chosen by $v_{i}>v_{0} . \beta_{2}$ is estimated using the Bootstrap method [21] as follows.

Step 1. Draw a sample from $1, \ldots, n$ with replacement and denoted by $i_{1}^{*}, \ldots, i_{n}^{*}$.

Step 2. Let $j=1, \ldots, n, q_{j}^{*}=q_{i^{*} j}$, and $v_{j}^{*}=v_{i^{*} j}$.

Step 3. Use $\left(q_{1}^{*}, v_{1}^{*}\right), \ldots,\left(q_{n}^{*}, v_{n}^{*}\right)$ to fit (2) and estimate the $\beta_{1 r}^{*}, \beta_{2 r}^{*}$.

Step 4. Repeat Steps $1 \sim 3, r=1, \ldots, R(R \geq 500)$.
Step 5. Use the $\beta_{2 r}^{*}(r=1, \ldots, R)$ to estimate the distribution of $\beta_{2}$.

Table 1 lists the results of hypothesis testing about the distribution of $\beta_{2}$. It is supposed that $\beta_{2}$ follows inverse Weibull distribution and log-normal and normal distribution, respectively. The results confirmed that $\beta_{2}$ follows inverse Weibull distribution and that the capacity is Weibull distributed:

$$
F_{C}(q)=1-\exp \left\{-\left[\frac{(q-900)}{1629}\right]^{21.57}\right\}
$$

\section{Conclusion}

In this paper, the traffic degradation concept was introduced and the degradation model was developed to describe the traffic breakdown process. In the model, the degradation rate is defined to explain the traffic breakdown occurring at various flow rate levels. Based on the degradation model, traffic breakdown probability and traffic capacity distribution were derived theoretically. The advantage of this approach is that only traffic flow rate and speed data are needed. It overcomes the difficulty of estimating traffic capacity without the observed capacity data. In this paper, it also validated that freeway capacity follows Weibull distribution.

However, there are still some problems to be solved in the future.

(1) Effect of geometric characteristics and traffic condition on the breakdown probability should be studied. Only by this, the research result may be applied generally.

(2) An acceptable breakdown probability value should be determined. This value reflects the balance between risk of breakdown and higher traffic volume. So, it is determined by the operating agency and questionnaires should be conducted.

(3) The definition of breakdown flow rate should be studied. Although, in this paper, the breakdown flow rate is defined as the flow rate observed immediately prior to breakdown, there are still some questions that need to be answered, such as the traffic flow that should be obtained for 5-minute, 10-minute, or 15minute time interval prior to breakdown. 


\section{Conflict of Interests}

The authors declare that there is no conflict of interests regarding the publication of the paper.

\section{Acknowledgment}

This work was supported by the National Key Basic Research Program of China under Grant no. 2012CB723303.

\section{References}

[1] Transportation Research Board, Highway Capacity Manual, National Research Council, Washington, DC, USA, 2000.

[2] A. Polus and M. A. Pollatschek, "Stochastic nature of freeway capacity and its estimation," Canadian Journal of Civil Engineering, vol. 29, no. 6, pp. 842-852, 2002.

[3] B. S. Kerner, "Three-phase traffic theory and highway capacity," Physica A: Statistical Mechanics and Its Applications, vol. 333, no. 1-4, pp. 379-440, 2004.

[4] F. L. Hall and A.-D. Kwaku, "Freeway capacity drop and the definition of capacity," Transportation Research Record, vol. 1320, pp. 91-97, 1991.

[5] R. Kühne, R. Mahnke, I. Lubashevsky, and J. Kaupužs, "Probabilistic description of traffic breakdowns," The Physical Review E: Statistical, Nonlinear, and Soft Matter Physics, vol. 65, no. 6, Article ID 066125, 2002.

[6] A. P. Tarko and Z. Tian, "Example analysis and handling of uncertainty in the highway capacity manual with consideration of traffic diversion," Transportation Research Record, vol. 1852, pp. 40-46, 2003.

[7] C.-Q. Shao, "Implementing estimation of capacity for freeway sections," Journal of Applied Mathematics, vol. 2011, Article ID 941481, 9 pages, 2011.

[8] L. Zhang and L. David, "Some properties of flows at freeway bottlenecks," in Proceedings of the Transportation Research Board 83rd Annual Meeting, National Research Council, Washington, DC, USA, 2004.

[9] M. R. Lorenz and L. Elefteriadou, "Defining freeway capacity as function of breakdown probability," Transportation Research Record, vol. 1776, pp. 43-51, 2001.

[10] W. Brilon, J. Geistefeldt, and M. Regler, "Reliability of freeway traffic flow: a stochastic concept of capacity," in Proceedings of the 16th International Symposium on Transportation and Traffic Theory, pp. 125-144, College Park, Md, USA, July 2005.

[11] K. Ozbay and E. E. Ozguven, "A comparative methodology for estimating the capacity of a freeway section," in Proceedings of the 10th International IEEE Conference on Intelligent Transportation Systems (ITSC '07), pp. 1034-1039, Seattle, Wash, USA, October 2007.

[12] O. Biham, A. A. Middleton, and D. Levine, "Self-organization and a dynamical transition in traffic-flow models," Physical Review A, vol. 46, no. 10, pp. R6124-R6127, 1992.

[13] J. Freund and T. Pöschel, "A statistical approach to vehicular traffic," Physica A: Statistical Mechanics and Its Applications, vol. 219, no. 1-2, pp. 95-113, 1995.

[14] A. Schadschneider, "Statistical physics of traffic flow," Physica A: Statistical Mechanics and Its Applications, vol. 285, no. 1, pp. 101-120, 2000.

[15] K. Nagel and M. Paczuski, "Emergent traffic jams," Physical Review E, vol. 51, no. 4, pp. 2909-2918, 1995.
[16] B. D. Greenshield, "A study of traffic capacity," Highway Research Board Proceedings, vol. 14, pp. 448-477, 1934.

[17] R. T. Underwood, Speed, Volume, and Density Relationships: Quality and Theory of Traffic Flow, Yale Bureau of Highway Traffic, New Haven, Conn, USA, 1961.

[18] J.-C. Lu, J. Park, and Q. Yang, "Statistical inference of a timeto-failure distribution derived from linear degradation data," Technometrics, vol. 39, no. 4, pp. 391-400, 1997.

[19] B. S. Kerner and S. L. Klenov, "Probabilistic breakdown phenomenon at on-ramp bottlenecks in three-phase traffic theory: congestion nucleation in spatially non-homogeneous traffic," Physica A: Statistical Mechanics and Its Applications, vol. 364, pp. 473-492, 2006.

[20] R. Dowling, A. Skabardonis, and D. Reinke, "Predicting impacts of intelligent transportation systems on freeway queue discharge flow variability," Transportation Research Record, vol. 2047, pp. 49-56, 2008.

[21] A. C. Davison and D. V. Hinkley, Bootstrap Methods and Their Application, Cambridge University Press, New York, NY, USA, 1997. 


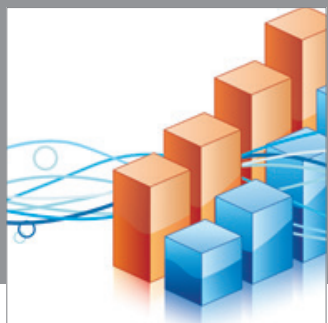

Advances in

Operations Research

mansans

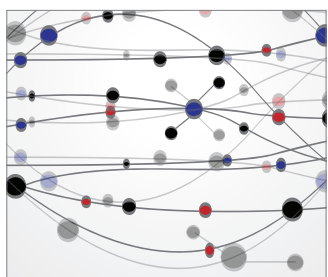

The Scientific World Journal
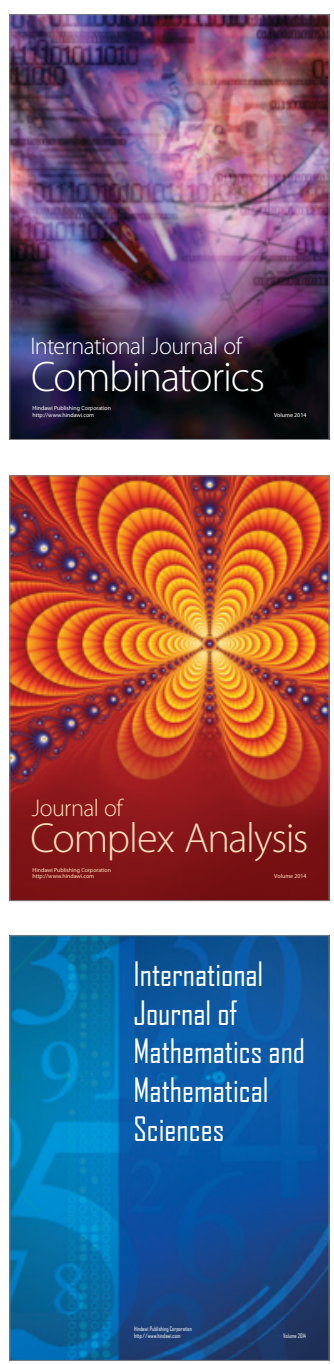
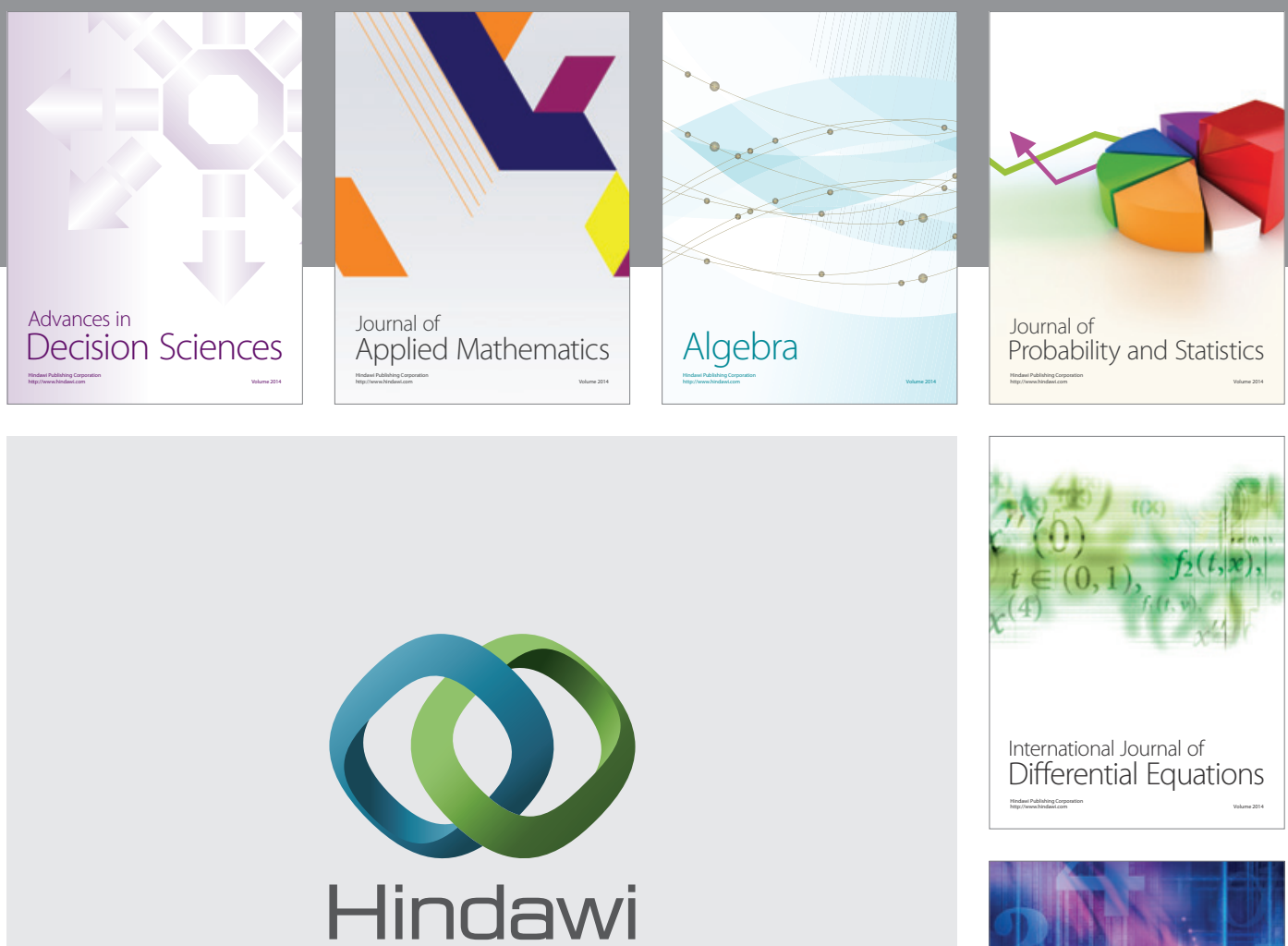

Submit your manuscripts at http://www.hindawi.com
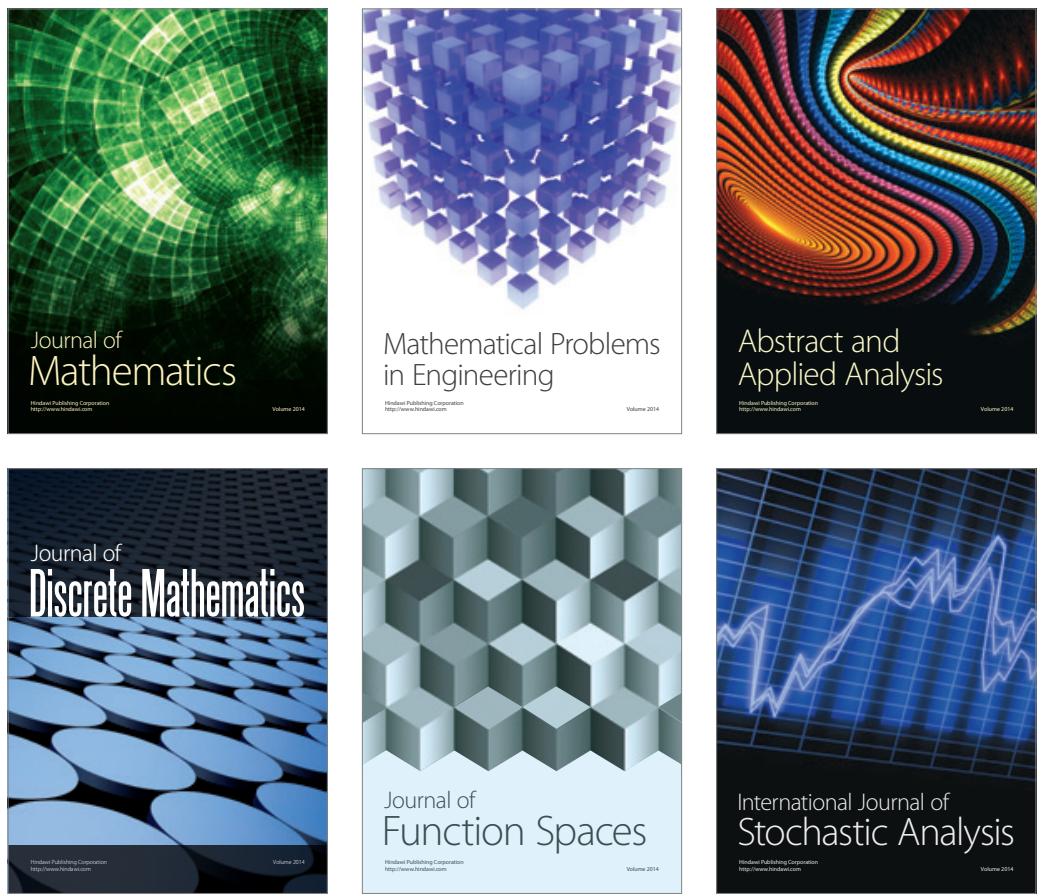

Journal of

Function Spaces

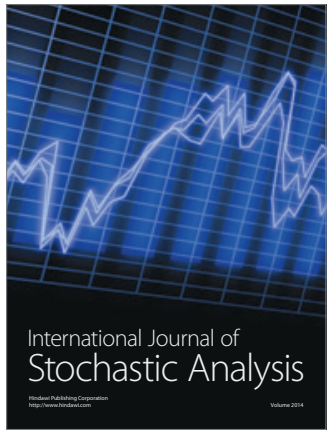

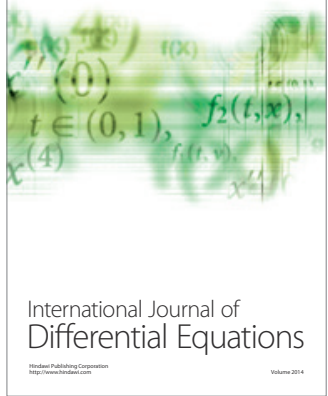
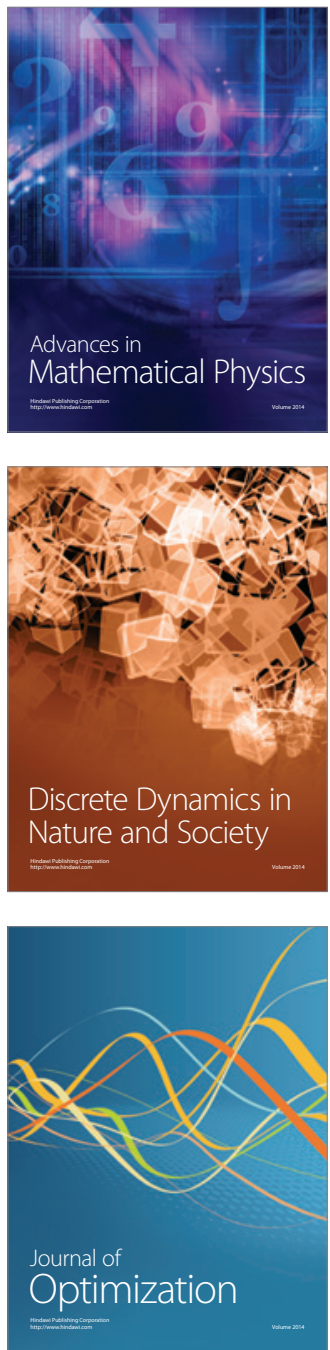